\title{
Conditions for the obtaining of tocopherols from deodorizing distillates of sunflower oil
}

\author{
Anastasiya Demydova ${ }^{1}$, Tamara Nosenko ${ }^{2}$, \\ Evgeniya Shemanska², Svitlana Molchenko'
}

1 - National Technical University «Kharkiv Polytechnic Institute», Kharkiv, Ukraine 2 - National University of Food Technologies, Kyiv, Ukraine

Keywords:

Tocopherol

Deodorizer

Distillate

Antioxidant

Oxidation

Absoption

\section{Article history:}

Received 20.08.2019

Received in revised

form 31.10.2019

Accepted 28.11.2019

Corresponding

author:

Anastasiya
Demydova
E-mail:
ademidova2016@
gmail.com

DOI: $10.24263 / 2310-$

1008-2019-7-2-4

\section{Abstract}

Introduction. A deodorizer distillate is a byproduct of oil deodoration, a natural source of tocopherols. The methods of obtaining a native antioxidants from a sunflower deodorizer distillate was investigated in this study.

Materials and methods. The sunflower deodorizer distillates from a local enterprise were used in this work. The unsaponificated fraction of deodorizer distillate was separated by saponification. The kinetics of sunflower oil oxidation with/without antioxidants is investigated by the volumetric method.

Results and Discussion. Deodorizer distillate increased sunflower oil induction period of oxidation by 1.5 times. Antioxidant activity of obtained concentrates was proved by increase of the induction period of oil oxidation almost three times as result of them addition in the amount of $50 \mathrm{mg}$ per $100 \mathrm{~g}$. Thus, saponification of deodorizer distillate gives possibility to obtain sufficiently effective inhibitor of oxidation.

It was shown that more effective way of antioxidants concentrating from a deodorizer distillates was to dissolve it in n-hexane with further adsorption of tocopherols on the activated carbon. Tocopherols were proposed to be eluted from activated carbon by m-xylene, after evaporation of which a concentrate of tocopherols was obtained. Obtained concentrate increased in the period of sunflower oil oxidation induction by 4.2 times when $50 \mathrm{mg}$ per $100 \mathrm{~g}$ of the antioxidant were added to the oil. The value of the rate constant of interaction of the peroxide radical with the inhibitor $\left(\mathrm{K}_{7}\right)$ for the obtained antioxidant was determined to be $10^{6} \mathrm{~mol} / 1 \cdot \mathrm{sec}$.

Conclusions. A method of obtaining tocopherol concentrates from sunflower oil deodorant was proposed. The efficacy of the obtained tocopherol concentrate as an antioxidant was proved. 


\section{Introduction}

It is known, that $\alpha$-tocopherol is one of the most active natural antioxidants. Tocopherols are registered as food additives: E306 (tocopherol mixture), E307 $(\alpha$ tocopherol), E308 ( $\gamma$-tocopherol) and E309 ( $\delta$-tocopherol) and are widely used both in food and in medicine $(\gamma, \delta$-forms have high vitamin activity) [1]. The term "vitamin E" or tocopherol is used for a large group of natural substances that have similar biological activity. In addition to the most common in nature $\alpha$-tocopherol, its 11 homologues and stereoisomers are known, all of them are currently isolated from vegetable oils or obtained synthetically [2]. Synthetic forms of tocopherols (labeled as "D, L" or "D") are approximately twice less active than natural [3]. Tokotrienols, which differ from tocopherols by the presence of unsaturated bonds in the carbohydrate chain, are also included to the "vitamin E" group. Subsequently, we will identify the total amount of tocopherols and tocotrienols as a tocopherol concentrate.

One of the main natural sources of tocopherols is vegetable oils. Their content in animal fats is very small and they are almost absent in fish fats. The amount of $\alpha$-tocopherol, for example, in unrefined sunflower oil, is approximately from 45 to $75 \mathrm{mg}$ per $100 \mathrm{~g}$ of oil, in soya oil $-75-170 \mathrm{mg}$ per $100 \mathrm{~g}$, in coconut oil $-3-8 \mathrm{mg}$ per $100 \mathrm{~g}$, etc. [4]. However, the amount of tocopherols is significantly reduced during process of refining oils, most of them are lost at the deodoration stage, where substances with specific smell and taste are distillated from oils at high temperatures and in deep vacuum. As a result, a by-product of deodorization (deodorizer distillate or deodistillate or scrubber oil) is formed, which contains predominantly fatty acids, triglycerides, mono-, diglycerides, sterols, tocopherols and others. shows The composition of deodorizer distillates from various vegetable oils are shown in Table $1[5,6,7]$.

Table 1

Composition deodorizer distillates different oils (g/100 g)

\begin{tabular}{|c|c|c|c|c|c|c|c|c|c|}
\hline \multirow[t]{2}{*}{ Lipid fraction } & \multicolumn{3}{|c|}{ Soy } & \multicolumn{2}{|c|}{ Corn } & \multicolumn{2}{|c|}{ Sunflower } & \multicolumn{2}{|c|}{ Rape } \\
\hline & 1 & 2 & 3 & 4 & 5 & 6 & 7 & 8 & 9 \\
\hline Squalene & 1,28 & 2,09 & 0,65 & 0,21 & 0,99 & 1 & 0,73 & 0,4 & 0,07 \\
\hline$\delta$ - tocopherol & 4,41 & 5,59 & 2,01 & 0,12 & 0,12 & - & - & 0,18 & 0,31 \\
\hline$\beta$ - tocopherol & 0,52 & 0,36 & 0 & 0,06 & 0,08 & - & - & 0,18 & 0,14 \\
\hline$\gamma$ - tocopherol & 10,73 & 11,26 & 4,96 & 1,09 & 2,75 & 0,07 & 0,3 & 2,48 & 2,33 \\
\hline$\alpha$ - tocopherol & 0,82 & 0,82 & 0,54 & 0,15 & 0,36 & 1,21 & 4,76 & 1,35 & 0,89 \\
\hline Campesterol & 5,06 & 5,66 & 1,91 & 0,84 & 1,67 & 0,45 & 1,58 & 4,37 & 2,93 \\
\hline Stigmasterol & 4,1 & 4,81 & 1,38 & 0,19 & 0,37 & 0,62 & 2,04 & - & 0,01 \\
\hline Phytosterol & 7,9 & 8,34 & 3,03 & 1,68 & 3,38 & 2,6 & 8,60 & 6,24 & 4,05 \\
\hline Phytosteride & 2,59 & 2,33 & 4,45 & 0,62 & - & 0,09 & 0,3 & 5,33 & 1,35 \\
\hline Monoglycerides & 1,24 & 1,93 & 1,85 & 0,04 & 0,13 & 0 & 0,86 & 1,42 & 2,11 \\
\hline Diglycerides & 2,7 & 3,79 & 8,06 & 0,54 & 1,26 & 0,66 & 1,89 & 3,85 & 3,87 \\
\hline Triglycerides & 2,7 & 3,79 & 8,06 & 0,54 & 1,26 & 0,66 & 1,89 & 3,85 & 3,87 \\
\hline Fatty acids (on $\mathrm{C}_{18: 1}$ ) & 33 & 32 & 73,8 & 81,2 & 77,1 & 70,82 & 39,2 & 39,2 & 42,8 \\
\hline
\end{tabular}

The quantitative composition of palm oil deodorizer distillate was established in [5]: fatty acids $81,7 \%$, acylglycerides $-14,4 \%$, squalene $-0,8 \%$, tocopherols $-0,5 \%$, sterols $0,4 \%$, other substances $-2.2 \%$. Typical composition of deodorizer distillate of olive oil 
according to [8] was characterized by high content of squalene $-28 \%$, free fatty acids $33.4 \%$, phytosterols $-4.6 \%$. Sunflower oil deodorizer distillate contains high content of $\alpha$ tocopherol.

It is also known that deodorizer distillates obtained during deodorization are characterized by higher content of tocopherols, sterols in comparison with a distillates obtained during distillation itself [10]. Thus, deodorizer distillates is a cheap source of valuable and useful substances such as tocopherols, sterols, squalene, and also fatty acids that have numerous industrial uses.

A number of basically different methods of tocopherol concentrate obtaining from deodorizer distillate are known. These methods include concentration of tocopherols by saponification of deodisystyllate, extraction of nonsaponificated substances, which contain also tocopherols [Pat. US20080015367, p. SU 1771474, p. EP0171009B1, p. RU 2485111 C1, p. US4550183 A, p. US4550183 A, p. US3335154A Pate. US2263550 A]. The disadvantage of this method is that besides tocopherols, all nonsaponificated substances are containing in deodistyllate concentrate reducing its value.

Another method of tocopherol concentration is the esterification of free fatty acids by lower monoatomic alcohols, followed by their extraction by polar and nonpolar solvents [Pat. US5660691 A, p. US5646311 A, p. US5487817 A, p. US4454329 A]. This methods is economically disadvantageous in comparison with other described methods, since the process of esterification is multistage and requires expensive equipment. Sometimes the combination of these two methods are using but still the esterification of free fatty acids is using [Pat. US7575767 B2].

Finally, the concentration of tocopherols by their adsorption on ion-exchange resins is using [Pat. US3122565 A]. This method is the most attractive, since it does not require multistage processing. However, selection of technological and economical best adsorbent is necessary for its most effective use.

Actually, deodorizer distillates are not used as a source of tocopherols. However, the capacity of domestic enterprises for the processing of sunflower oil is significant, and therefore the development of technology for obtaining the valuable products from a deodorizer distillates is a significant task.

The known methods for producing tocopherols from deodorizer distillates are multistage, require the high temperatures and deep vacuum, that is, expensive equipment and high energy consumption.

The objective of this work was to develop the simple and cheap method for obtaining of native antioxidant - tocopherol concentrate from sunflower deodorizer distillates including as few stages as possible, not requiring high temperatures and providing high antioxidant activity.

\section{Materials and methods}

Sunflower deodorizer distillates, activated carbon RAN-200 were used for tocopherols obtaining. Refining dedorizated winterizated sunflower oil was used as model substance for determination of tocopherol concentrates antioxidant activity.

\section{Proximate analyses of oxidation}

Content of initial oxidation products was estimated according to peroxide value. Content of aldehydes and ketones (secondary oxidation products) was investigated by the method of determination of carbonyl compounds. 


\section{Separation of nonsaponificated and saponificated substances of deodorizer distillates}

Saponification of deodorizer distillate was carried out by heating it to $75^{\circ} \mathrm{C}$ and adding a water-alcohol $0.5 \mathrm{~mol} / \mathrm{dm}^{3}$ solution of potassium hydroxide. Saponification was performed during 1 hour at constant stirring. After this, the nonsaponificated substances were extracteded from the reaction mixture with n-hexane followed by distillation of the solvent. [11].

\section{Recovering the tocopherols from deodorant distillates}

The separation of tocopherols on activated carbon was carried out according to the following procedure: the deodorant distillate was dissolved in n-hexane in the ratio 1:1.5 1:5, respectively. The resulting solution was passed twice through a column filled with a layer of activated carbon. The tocopherols were eluated from carbon column by m-xylene and m-xylene was distilled from eluate at $139.1{ }^{\circ} \mathrm{C}$ and obtained residue was a tocopherol concentrate.

\section{Determination of sunflower oil oxidation kinetics}

The kinetics of sunflower oil oxidation was investigated by the volumetric method [12]. The mesuaring was carried out at $70^{\circ} \mathrm{C}$ under conditions of initiated oxidation, that is, due to the thermal decomposition of the solution of AIBN (azoizobutyronitrile), concentration of AIBN was $2 \mathrm{Mmol}$ for all samples, that provided the constant oxidation rate initiation. The value of the induction period $(\tau, \mathrm{c})$ was determined by graphical method. This value is equal to the segment of the abscissa, which is cut off by a perpendicular from the point of intersection of the tangents to the kinetic curve.

The fixed amount of antioxidants $-2 \mathrm{mg} / 100 \mathrm{~g}$ was added to the samples of oil. Antioxidant concentration ([InH], mol/L) was determined according to equation [12]:

$$
[\operatorname{InH}]=\frac{1 \times[A I B N] \times\left(1-e^{-K_{p} \times \tau}\right)}{f}=0,48 \times[A I B N] \times\left(1-0,9999^{\tau}\right),
$$

where [AIBN] - initiator concentration, mol/L; $\mathrm{K}_{\mathrm{p}}-$ constant of rate of initiator decomposition at $70{ }^{0} \mathrm{C}\left(3,9 \cdot 10^{-5} \mathrm{sec}^{-1}\right) ; 1 / \mathrm{f}=0.48$ (1- radical quantity under destruction of one initiator molecule), $\tau$ - determined induction period, sec.

\section{Determination of the rate constant of the oxidation chains termination}

The antioxidant activity of obtained tocopherol concentrate was estimated as a rate constant of the oxidation chains decay $\left(\mathrm{K}_{7}\right)$ [12]. The $0.3 \mathrm{~g}$ tocopherol concentrate obtained were dissolved in $50 \mathrm{ml}$ of xylene, and $0.1 \mathrm{ml}$ of solution was added to $5 \mathrm{ml}$ of cumene (the concentration of tocopherols approximately equal to their concentration in refined sunflower oil). The rate of oxidation of this solution was determined under different concentration of the initiator of oxidation (from $10^{-3}$ to $10^{-2} \mathrm{~mol} / \mathrm{L}$ ).

The reaction rate constant was calculated according to:

$$
K_{p} / K_{7}=\operatorname{tg} \alpha \times \Delta V /[R H] \times v,
$$

where $\operatorname{tg} \alpha$ - tangent of the slope angle of line, which reflects the dependence of the oxidation rate on the rate of initiation of cumene enriched with tocopherols, $\Delta \mathrm{V}$ - volume of $1 \mathrm{~mm}^{3}$ oxygen, under normal condition $\Delta V=4,09 \times 10^{-5} \mathrm{Mol} / \mathrm{L} ;[R H]$ - hydrocarbon concentration; $v$ - sample volume, $1 ; K_{p}-$ rate constant of the cumene oxidation, at $70{ }^{\circ} \mathrm{C} K_{p}=3,19 \mathrm{~L} / \mathrm{mol} \cdot \sec [12]$. 


\section{Results and discussion}

Influence of sunflower deodorizer distillate and its unsaponificated fraction on the induction period of sunflower oil oxidation

The sunflower deodorizer distillate and its unsaponificated fraction were tested as inhibitors of oxidation. The kinetics of oxidation of sunflower refined oil were analyzed with the addition of $0.05 \%$ deodorizer distillate or unsaponificated substances. The results are shown in the Table 2.

Table 2

Parameters of sunflower refined oil oxidation under addition of deodorizer distillate and unsaponificated substances

\begin{tabular}{|l|c|c|}
\hline Sunflower refined oil sample & $\begin{array}{c}\text { Induction period } \\
\text { of oxidation, sec }\end{array}$ & $\begin{array}{c}\text { Inhibitor } \\
\text { concentration, mol/L }\end{array}$ \\
\hline Sunflower refined oil & 1320 & $1,1 \times 10^{-4}$ \\
\hline $\begin{array}{l}\text { Sunflower refined oil + deodorizer } \\
\text { distillate }\end{array}$ & 1980 & $1,7 \times 10^{-4}$ \\
\hline $\begin{array}{l}\text { Sunflower refined oil + } \\
\text { unsaponificated substances }\end{array}$ & 4200 & $3,2 \times 10^{-4}$ \\
\hline
\end{tabular}

Unsaponificated fraction of deodorizer distillate increases sunflower oil induction period by 2.9 times, while deodorizer distillate itself only by 1.5 times. Thus, saponification of deodorizer distillate gives possibility to obtain sufficiently effective inhibitor of oxidation. Also, when applying this approach, a by-product - soap, which is also a commodity product [13], is produced.

For further increase of tocopherol concentration and subtiantly the antioxidant activity of concentrate the method of tocopherols recovering using their absorption on activated carbon was investigated.

\section{Effect of antioxidant, obtained using activated carbon, on the oxidation kinetics of sunflower oil}

The concentrate of tocopherols, obtained after purification on the carbon column, when added $0,05 \%$ to sunflower oil extended its induction period of oxidation from $1320 \mathrm{sec}$ to $5600 \mathrm{sec}$ (4.2 times, Figure 1). These data proved that proposed method of tocopherols recovering from unsaponificated fraction of deodorizer distillate is available for purification and concentration of tocopherols.

\section{Antioxidant activity of the obtained tocopherol concentrate}

For estimation of antioxidant activity of the obtained tocopherol concentrate the rate constant of interaction of peroxide radicals with the inhibitor $\left(\mathrm{K}_{7}\right)$ was measured. Cumene was chosen as oxidation medium, an effective reducing agent whose oxidation rates are well studied and known [12]. Oxidation kinetics of cumene in the presence of obtained tocopherol concentrate under different concentration of AIBN are shown in Figure 2. 


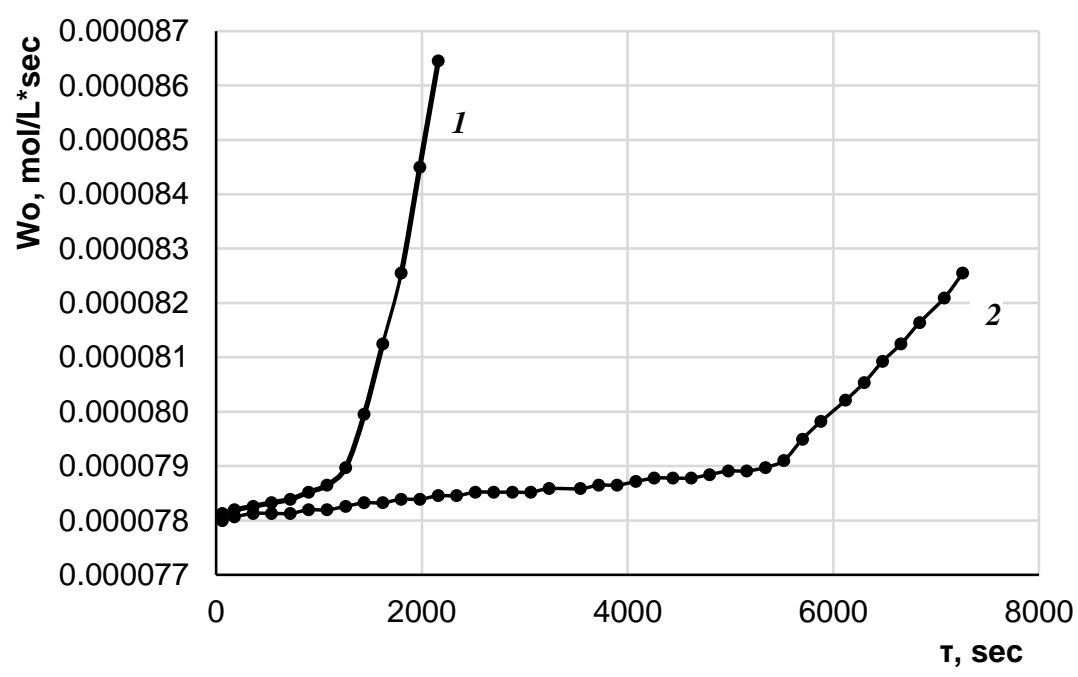

Figure 1. Oxidation kinetics of sunflower oil:

1 - sunflower refined oil, 2 - sunflower refined oil with addition of tocopherols concentrate, purified by carbon absorption

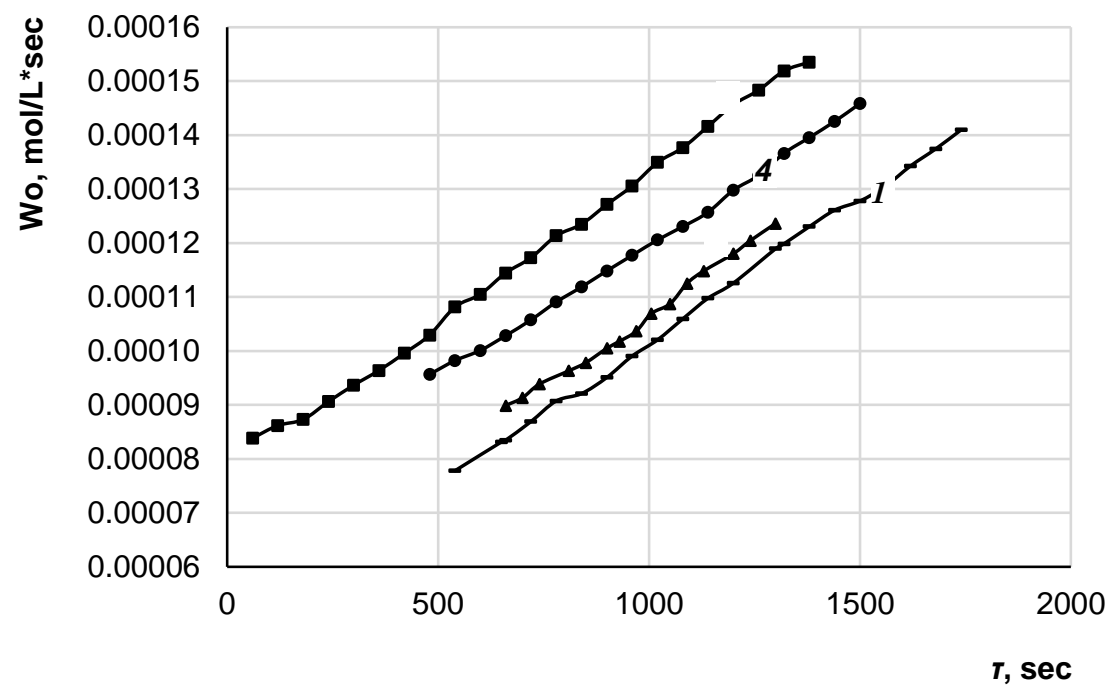

Figure 2. Kinetics of cumene oxidation in the presence of obtained antioxidant concentrate at different concentrations of AIBN:

$1-4 \cdot 10^{-3} \mathrm{~mol} / \mathrm{L}$ AIBN, $2-5 \cdot 10^{-3} \mathrm{~mol} / \mathrm{L}, 3-6 \cdot 10^{-3} \mathrm{~mol} / \mathrm{L}, 4-8 \cdot 10^{-3} \mathrm{~mol} / \mathrm{L}$ 
The rate constant of interaction of peroxide radicals with the inhibitor $\left(\mathrm{K}_{7}\right)$ was determined graphically as the value of a segment on the $\mathrm{Y}$ axis, cutted by the line of dependence between the rate of oxidation and the rate of initiation of cumene in the presence of antioxidant (Figure 3).

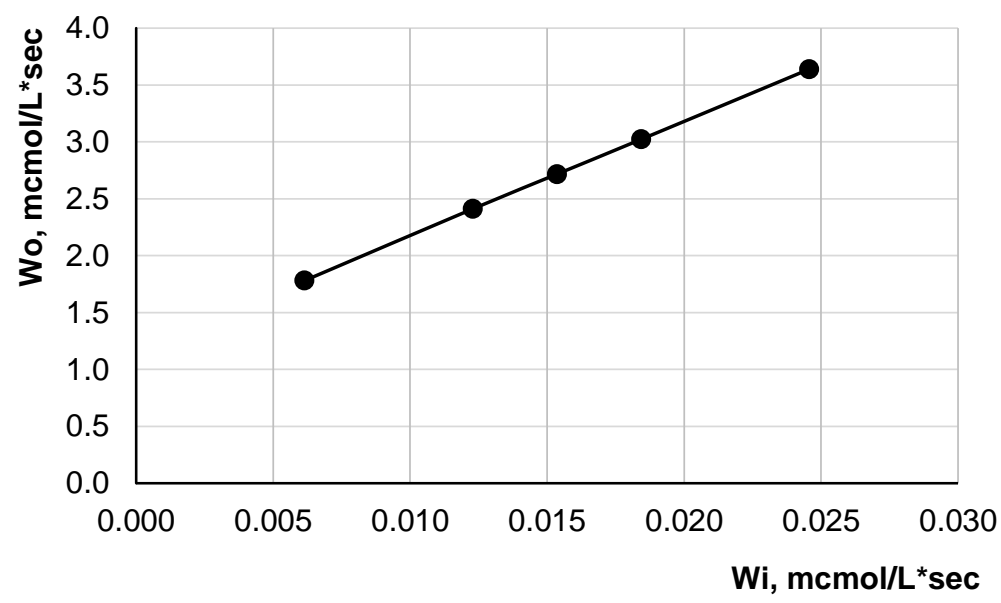

Figure 3. Dependence of the oxidation rate on the initiation rate of cumene in the presence of obtained antioxidant

The rate constant of the reaction between the inhibitor and the peroxide radical was also determined according to equation (2) and was $\mathrm{K}_{7}=0.94 \cdot 10^{6} \mathrm{~L} / \mathrm{mol} \cdot \mathrm{sec}$. The obtained value approximately agreed with the known $\mathrm{K}_{7}$ constants for tocopherols [12], that is, the obtained oxidation inhibitor can be recommended as a tocopherol concentrate.

\section{Content of oxidation products in deodorizer distillate and isolated antioxidant}

Content of oxidation products in different antioxidant fraction was determined in obtained concentrates (Table 3). Content of peroxides and carbonic substances was the lowest in tocopherols concentrate, obtained by absorption purification, and highest in the initial sunflower deodorizer distillate.

Thus developed method of tocopherols concentrate obtaining includes the next steps:

(A) the deodorizer distillate is dissolved in $n$-hexane in a ratio of $1: 1.5$ to $1: 3$ in order to reduce its viscosity and improve the contact of tocopherols with activated carbon (in another case it is enough to warm distillate up to $5^{\circ} \mathrm{C}$ of its melting point);

(B) the obtaining solution is passed through a layer of activated carbon, on which the tocopherols are absorbed, and most of other components are passed;

(C) to remove tocopherols from the adsorbent polar solvent $\mathrm{m}$-xylene in the ratio $\mathrm{m}$ xylene:deodorizer distillate from 1:1,5 to 1:3 is passed through the layer of activated carbon;

(D) m-xylene is evaporated from the tocopherols concentrate at $139.1{ }^{\circ} \mathrm{C}$. 
Content of oxidation products in deodorizer distillate and isolated antioxidant

\begin{tabular}{|l|c|c|}
\hline \multicolumn{1}{|c|}{ Sample } & $\begin{array}{c}\text { Peroxide value, } \\
\text { mmol 1/2 O/kg }\end{array}$ & $\begin{array}{c}\text { Carbonyl value, } \\
\mathbf{m g ~ K O H / g}\end{array}$ \\
\hline Sunflower deodorizer distillate & 31,5 & 86,9 \\
\hline $\begin{array}{l}\text { Unsaponificated substances of sunflower } \\
\text { deodorizer distillate }\end{array}$ & 15,5 & 56,1 \\
\hline $\begin{array}{l}\text { Tocopherols concentrate, obtained by } \\
\text { absorption purification }\end{array}$ & 10,0 & 38,5 \\
\hline
\end{tabular}

Using of the developed method for tocopherols concentrate obtaining gives the following advantages compared to the known technology: it does not require a multi-stage approach [14], which usually includes the distillation of deodorizer distillates and esterification of fatty acids and other components. It gives the opportunity to receive not only a tocopherol concentrate as a commercial product, but also a mixture of fatty acids and sterols, which can be used as a suitable raw material for the further removal of sterols and fatty acids. Adsorbent and solvents can be completely regenerated, that reduces the cost of the final product.

In addition the content, of benzpirene in the obtaining concentrate of tocopherols is significantly reduced compared to the content of benzpirene in the initial deodorizer distillate because of limited solubility of benzpirene in hexane. However, in case of the presence of this toxic substance in the final concentrate of tocopherols such samples can be recommended to be used for technical purposes. For example, in the production of lubricant and cooling products based on vegetable oils, antioxidants need to be used [15]. Lubricant and cooling products are traditionally obtained from the mineral oils. However, such materials are one of the main pollutants of the environment, and it is promising to replace them with vegetable oils [16].

The obtained concentrate of tocopherols also contains phytosterols, which are valuable biologically active substances. The tocopherols concentrate obtained from deodorizer distillate with a low content of benzpirene can be recommended for use in the food production.

\section{Conclusions}

1. Cost-effective method of obtaining a tocopherols concentrate from a by-product of oil refining - sunflower deodorizer distillate was developed. Efficiency as an antioxidant of the obtained concentrate is proved.

2. The scientific novelty of the work is to study the antioxidant effect of the obtained tocopherol concentrate. The reaction rate constant between the inhibitor and the peroxide radical was $\mathrm{K}_{7}=0.94 \cdot 10^{6} \mathrm{~L} / \mathrm{mol} \cdot \mathrm{s}$, which proves the effectiveness of using the obtained tocopherol concentrate as an antioxidant.

3. The prospective methods of tocopherols extracting include the idea of saponification of deodorizer distillate at the first stage and the concentration of tocopherols on the activated carbon at the second stage. In this case most of the deodorizer distillate is separated at the first stage, that reduces the required amount of activated carbon and allows to obtain a product with a high content of tocopherols. 


\section{References}

1. Fereidoon Shahidi (2016), Tocopherols and Tocotrienols in Common and Emerging Dietary Sources: Occurrence, Applications, and Health Benefits, Int. J. Mol. Sci., 17(10), pp. 1745-1774.

2. Nadirov N.K. (1991), Tokoferoly $i$ ih ispolzovanie $v$ medicine i selskom hozjajstve, Moscow.

3. Sanjay Kumar Gupta, Munagala Gayatri Ramya, Rajesh Akki (2013), Development and validation of new RP-HPLC methods for stability study of fat soluble vitamins, International Journal of Pharmacy and Pharmaceutical Sciences, 5, 3, pp. 71-75.

4. Sizova N.V. (2013), Opredelenie tokoferolov kak lipidnyh antioksidantov v rastitelnyh maslah i zhivotnyh zhirah, Himija rastitelnogo syrja, 1, pp. 157-163.

5. Top A. (2010) Production and utilization of palm fatty acid distillat, PFAD, Lipid Technol., 22, pp. 11-13.

6. Carmona M.A., Jiménez-Sanchidrián C., Ruiz J.R. (2015), Recent Developments in Phytosterol Recovery from Oil Deodorizer Distillates, Curr. Nutr. Food Sci. 11, pp. 4-10.

7. Naz S., Sherazi S.T.H., Talpur F.N., Kara H., Sirajuddin, Khaskheli A.R. (2014), Chemical characterization of canola and sunflower oil deodorizer distillates, Pol. J. Food Nutr. Sci., 64, pp. 115-120.

8. Bondioli P. (2013), Squalene recovery from olive oil deodorizer distillate, J. Am. Oil Chem. Soc., 70, pp. 763-766.

9. Verleyen T., Verhe R., Garcia L., Dewettinck K.,Huyghebaert A., De Greyt W. (2001), Gas chromatographic characterization of vegetable oil deodorization distillate, J Chromatogr, A 921, pp. 277-285.

10. Yang H., Yan F., Wu D., Huo M., Li J., Cao Y., Jiang Y. (2010), Recovery of phytosterols from waste residue of soybean oil deodorizer distillate, Bioresour. Technol., 101, pp. 1471-1476.

11. Cici Maarasyid, Ida Idayu \& Eko Supriyanto (2014) Potential Source and Extraction of Vitamin E From Palm-Based Oils: A Review, Jurnal Teknologi (Sciences \& Engineering) 69:4 (2014), 43-50.

12. Ushkalova V.N. (1988), Stabilnos lipidov pishhevyh produktov, Moscow, $152 \mathrm{p}$.

13. Lienda A. Handojo, Antonius Indarto, Dian Shofinita, Anggina Meitha, Rakhmawati Nabila, Harry Triharyogi, Leonardus Kevin (2018), Calcium soap from palm fatty acid distillate for ruminant feed: The influence of water temperature, IOP Conf. Series: Earth and Environmental Science, 141, 1.

14. Malekbala M. R., Soltani S. M., Hosseini S., Eghbali Babadi F., \& Malekbala R. (2015), Current technologies in the extraction, enrichment and analytical detection of tocopherols and tocotrienols: A review. Critical Reviews in Food Science and Nutrition, 57(14), pp. 2935-2942.

15.Zhixin Xiao, Chen Shi, Daheng Mao (2008), Effect of tocopherol on antioxygenic properties of green lubricating oil, Journal of Wuhan University of Technology-Mater. Sci. Ed. June, 23, 3, pp. 289-292.

16. Panchal T.M., Patel A., Chauhan D.D., Thomas M., \& Patel J.V. (2017), A methodological review on bio-lubricants from vegetable oil based resources. Renewable and Sustainable Energy Reviews, 70, pp. 65-70. 